\title{
CUIDADO AL PERSONAL DE ENFERMERÍA DESDE LA PERSPECTIVA DEL SUPERVISOR EN EL CONTEXTO HOSPITALARIO
}

\author{
NURSING STAFF CARE FROM A SUPERVISOR'S PERSPECTIVE IN THE \\ HOSPITAL SETTING
}

\section{CUIDADOS DO PESSOAL DE ENFERMAGEM NA PERSPECTIVA DE UM SUPERVISOR NO AMBIENTE HOSPITALAR}

\author{
Luis Arturo Pacheco-Pérez* \\ Karla Judith Ruiz-González** \\ Milton Carlos Guevara-Valtier ${ }^{* * *}$ \\ Juana Mercedes Gutiérrez-Valverde**** \\ Rosario Edith Ortiz-FÉLIX*****
}

\section{RESUMEN}

Objetivo: Describir el cuidado al personal de enfermería desde la perspectiva del supervisor en el contexto hospitalario. Material y Método: Estudio exploratorio con aproximación cualitativa en el que se empleó el diseño sistemático de la Teoría Fundamentada; la población se conformó por supervisores de enfermería en dos hospitales públicos de segundo nivel en la ciudad de Chihuahua, México; la recolección de datos se llevó a cabo durante agosto y septiembre de 2017 a través de un muestreo teórico, respetando los aspectos éticos de investigación. Resultados: Con diez participantes, hasta llegar a la saturación de datos, predominó el sexo femenino (7), en su mayoría casados (6) y con estudios de posgrado (6). Surgieron tres categorías: 1) Cuidado desde la perspectiva del supervisor de enfermería; 2) Interacción entre el supervisor de enfermería y sus compańeros de trabajo y 3) Desarrollo profesional como parte del cuidado al supervisor de enfermería. Conclusiones: La mayoría conceptualiza el cuidado como una actividad dirigida a otros pero no a sí mismos. La comunicación efectiva y estilo de liderazgo desempeñan una función importante para la interacción del supervisor de enfermería con el personal de enfermería operativo y sus directivos, pero estos atributos no se perciben de manera adecuada por ellos mismos.

Palabras clave: Supervisión de Enfermería; Atención de Enfermería; Liderazgo; Percepción; Desarrollo de Personal.

*Doctor en Ciencias de Enfermería. Departamento de Ciencias de la Salud, Universidad de Sonora. Cd. Obregón, México. ORCID: https://orcid.org/0000-0002-3765-5559 Email: luispachecoperez@gmail.com

**Doctora en Administración. Supervisión de Enfermería, Hospital General “Dr. Salvador Zubirán Anchondo”, Chihuahua, México. ORCID: https://orcid.org/0000-0002-9919-8103 Email: kar_ruglez@hotmail.com Autora de correspondencia.

***Doctor en Educación. Facultad de Enfermería, Universidad Autónoma de Nuevo León. Monterrey, México. ORCID: https:// orcid.org/0000-0002-0806-3706 Email: carlos_valtier7@hotmail.com

****Doctora en Ciencias de Enfermería. Facultad de Enfermería, Universidad Autónoma de Nuevo León. Monterrey, México. ORCID: https://orcid.org/0000-0001-9506-5947 Email: juanymeche@yahoo.com.mx

*****Doctora en Ciencias de Enfermería. Facultad de Enfermería Mochis, Universidad Autónoma de Sinaloa. Los Mochis, México. ORCID: https://orcid.org/0000-0002-5827-3218 Email: edithof51@hotmail.com 


\section{ABSTRACT}

Objective: To describe nursing staff care from the supervisor's perspective in the hospital setting. Material and Methods: Exploratory study with a qualitative approach using the systematic design of the Grounded Theory. The population consisted of nursing supervisors in two secondary care public hospitals in the city of Chihuahua, Mexico; data collection was carried out during August and September 2017 through theoretical sampling, respecting ethical aspects of research. Results: With ten participants until reaching data saturation, female sex predominated (7), mostly married (6) and with postgraduate studies (6). Three categories emerged from this study: 1) Care from the nursing supervisor's perspective; 2) Interaction between the nursing supervisor and their coworkers and 3) Professional development as part of the nursing supervisor care. Conclusions: Most supervisors conceptualize care as an activity aimed at others, but not at themselves. Effective communication and leadership style play an important role in the interaction between nurse supervisors with operational nursing staff and their managers, but these attributes are not properly perceived by themselves.

Key words: Nursing Supervision; Nursing Care; Leadership; Perception; Staff Development.

\section{RESUMO}

Objetivo: Descrever os cuidados do pessoal de enfermagem da perspectiva do supervisor no contexto hospitalar. Material e Método: Estudo exploratório com abordagem qualitativa usando o desenho sistemático da teoria fundamentada ou Grounded Theory. A população consistiu de gerentes de enfermagem em dois hospitais públicos de cuidados secundários na cidade de Chihuahua, México; a coleta de dados foi realizada nos meses de agosto e setembro de 2017 por amostragem teórica, respeitando os aspectos éticos da pesquisa. Resultados: com dez participantes até atingir a saturação de dados, predominando o sexo feminino (7), a maioria casada (6) e com estudos de pós-graduação (6). Três categorias emergiram deste estudo: 1) percepção do gerente de enfermagem sobre o cuidado; 2) interação entre o gerente de enfermagem e colegas de trabalho e 3) desenvolvimento profissional como parte do atendimento do gerente de enfermagem. Conclusóes: A maioria dos enfermeiros gerentes conceitua o cuidado como uma atividade voltada para os outros, mas náo para si mesmos. O estilo eficaz de comunicação e liderança desempenha um papel importante na interação do gerente de enfermagem com a equipe de enfermagem e seu diretor executivo, mas esses atributos não são percebidos adequadamente por eles mesmos.

Palavras-chave: Supervisão de Enfermagem; Cuidados de Enfermagem; Liderança; Percepção; Desenvolvimento de Pessoal.

Fecha de recepción: 07/04/2020

Fecha de aceptación: 03/02/2021

\section{INTRODUCCIÓN}

El supervisor de enfermería (SE), en adelante entiéndase supervisor o supervisora, es parte fundamental de las instituciones de salud y una de sus funciones es influir en el crecimiento profesional del personal operativo, para transmitir los valores y misión de la institución, fomentando un agradable ambiente laboral, además de mejorar la interacción entre el equipo multidisciplinario de salud, promoviendo mejores prácticas para otorgar cuidado de calidad ${ }^{(1)}$. Lo anterior requiere de profesionales con capacidad para adaptarse a un ambiente en constante cambio, en donde se necesita implementar estrategias basadas en la evidencia científica. Lo anterior implica que el SE debe tener conocimiento sobre los diferentes roles y actividades que desempeña el personal operativo para cumplir sus objetivos, a través del manejo eficiente de recursos, valiéndose de tres dimensiones: ciencia, liderazgo y $\operatorname{arte}^{(2,3)}$.

Durante el desempeño laboral del SE emergen situaciones que pueden influir en su decisión de continuar en el puesto de supervisor o renunciar 
a él. Entre esas situaciones destacan los problemas internos relacionados con la mala comunicación entre el equipo de trabajo o la falta de capacitación en actividades gerenciales, y se han acostumbrado a convivir con los conflictos que se presentan como parte de la rutina de trabajo ${ }^{(4)}$. Por ello es importante favorecer su asertividad con el personal, ya que las funciones gerenciales requieren de un profesional con habilidades de inteligencia emocional y educación avanzada, además de un estilo de liderazgo que motive a las personas a su cargo $^{(5)}$.

Diversos estudios hacen referencia a problemas que experimenta el personal de enfermería en la interacción con el SE, por ejemplo: mencionan que no existe una adecuada retroalimentación a sus actividades, que la supervisión es muy estricta y que junto con maximizar cualquier problema que se presente, no se hace de manera privada. También es común que no se le reconozca el cumplimiento satisfactorio de sus funciones, además de que no notifica a tiempo ni en forma adecuada la información referida a la institución, por lo que se sugiere que el SE debe contar con la habilidad de mejorar la comunicación entre los distintos departamentos y jerarquías institucionales para favorecer el compromiso laboral ${ }^{(6-8)}$.

De acuerdo con lo anterior, el ambiente laboral tiene repercusión directa en el trato del SE con el personal operativo, relación que está influenciada por el estilo de liderazgo, equipo de trabajo y su habilidad para solucionar conflictos, sin embargo, el SE también puede ser víctima de violencia laboral, la mayoría de las veces propiciado por personal a su cargo, pares supervisores y superiores ${ }^{(9-12)}$.

El personal que se desempeña como SE en hospitales públicos, en México, recibe el nombramiento por su capacidad para ser resolutivos, proactivos y asertivos, además del conocimiento que se tiene de las diferentes áreas hospitalarias y del manejo del capital humano; sin embargo, no en todas las instituciones del país el SE cuenta con reconocimiento oficial y, por lo tanto, no perciben la remuneración de acuerdo con las funciones, lo que ocasiona una constante rotación de personal en el área, al no sentirse cuidados por su institución y su percepción del ambiente laboral puede ser negativa.

Considerando contribuir con las investigaciones del tema en el contexto mexicano, el presente estudio tuvo por objetivo describir el cuidado al personal de enfermería, desde la perspectiva del $\mathrm{SE}$ en el contexto hospitalario, tomando como base el ambiente laboral y desarrollo profesional en instituciones públicas de segundo nivel de atención.

\section{MATERIAL Y MÉTODO}

Diseño: Estudio con aproximación cualitativa en el que se empleó el diseño sistemático de la Teoría Fundamentada, que permite la construcción de categorías y subcategorías para describir la realidad desde la perspectiva de los participantes ${ }^{(13)}$. Con este diseño se pretende comprender el significado de las experiencias y cómo las viven las personas.

Participantes: La población diana de este estudio estuvo conformada por 26 supervisores de enfermería, en dos hospitales públicos de segundo nivel de atención en la ciudad de Chihuahua, México (Tabla 1). Su función principal era supervisar el cuidado directo proporcionado a los pacientes y la gestión de los recursos de las áreas a su cargo. Cada supervisor atendía 4 o 5 servicios, dividiéndose por áreas críticas, quirúrgicas y hospitalización general, donde, en promedio, eran 20 personas del área operativa a su cargo, con estudios desde el nivel técnico hasta posgraduados.

Para la selección de los participantes se empleó el muestreo teórico, porque permite incluir a personas que están inmersas de manera directa en el fenómeno de estudio, de acuerdo con la información que se va obteniendo ${ }^{(14)}$. Se buscó a participantes que cumplieran con las siguientes características (criterios de inclusión): 1) personal supervisor de enfermería con al menos 5 años de experiencia en el puesto y 2) que contaran con al menos 5 años de experiencia como personal operativo antes de ser supervisores a objeto de que tuviera conocimiento sobre la mayoría de las áreas y que conocieran el trabajo del personal operativo.

Recolección de datos: Se llevó a cabo durante agosto y septiembre de 2017 por la investigadora principal, que cuenta estudios profesionales de enfermería, doctorado en administración, 10 años de experiencia clínica y de investigación. Previamente realizó una estancia de observación participante en cada institución con duración de tres semanas, permitiendo establecer la confianza 
necesaria para la obtención de información.

Se realizó una entrevista semiestructurada, que permite incluir más preguntas para enriquecer la información ${ }^{(11)}$, hasta llegar a la saturación de los datos al obtener información repetida, por lo que el grupo de participantes se conformó por 10 personas que cumplieron los criterios de inclusión; ninguno rechazó participar. Las entrevistas se desarrollaron en un aula de enseńanza para evitar interrupciones, se llevaron a cabo privadamente con cada participante dentro de su horario laboral para no interferir con su trabajo, tuvieron una duración entre 20 y 40 minutos y fueron grabadas. Se elaboró un guión con las siguientes preguntas: 1) ¿Para usted, qué es el cuidado y cómo lo contextualiza en su quehacer?; 2) ¿Considera que en su institución cuidan del supervisor de enfermería?; 3) ¿Cómo fomenta la institución su desarrollo profesional? y 4) ¿Cómo es el ambiente laboral en su institución?

Codificación: Cada participante se identificó con la letra mayúscula $\mathrm{P}$ seguido de un número, desde 01 al 10. Se transcribieron las entrevistas y se realizó el análisis de toda la información obtenida, para luego codificar de manera abierta, axial y selectiva, creando categorías y subcategorías desde la perspectiva de los autores.

Para asegurar el rigor científico, se respetaron los siguientes principios: dependencia, credibilidad, transferencia y confirmabilidad ${ }^{(13)}$. Lo anterior se logró después de analizar la información detalladamente, incluyendo anotaciones sobre lenguaje no verbal, expresiones verbales, énfasis en palabras, entre otras. La información recopilada se analizó nuevamente con apoyo de dos investigadores cualitativos para ahondar en los datos y se validó con los participantes al mostrar las transcripciones y resultados finales.

Consideraciones éticas: La investigación fue aprobada por las autoridades de la Facultad de Contaduría y Administración de la Universidad Autónoma de Chihuahua y de las dos instituciones participantes, además se apegó a lo estipulado en el Reglamento de la Ley General de Salud en Materia de Investigación para la Salud ${ }^{(15)}$. Se entregó a cada participante un consentimiento informado y se comunicó su derecho a renunciar al estudio o retirar su información y grabaciones en cualquier momento, además de la confidencialidad de la información obtenida y el manejo de datos personales.

\section{RESULTADOS}

En el estudio participaron 10 SE, predominando el sexo femenino (7), en su mayoría casados (6) y con estudios de posgrado (6) (Tabla 1). Surgieron tres categorías que en conjunto integran la percepción del supervisor de enfermería (SE) sobre el cuidado al personal en el contexto hospitalario: 1) Cuidado desde la perspectiva del supervisor de enfermería, 2) Interacción entre el supervisor de enfermería y sus compañeros de trabajo y 3) Desarrollo profesional como parte del cuidado al supervisor de enfermería.

Tabla 1. Características del Supervisor de Enfermería (SE) en dos hospitales públicos, ciudad de Chihuahua, México, 2017.

\begin{tabular}{l|c|c|c|c|c}
\hline Participante & Sexo & Edad & Estado civil & Turno & Nivel de estudio \\
\hline PE1 & Masculino & 34 & Casado & Matutino & Posgrado \\
PE2 & Femenino & 33 & Soltera & Matutino & Posgrado \\
PE3 & Femenino & 49 & Casada & Vespertino & Licenciatura \\
PE4 & Femenino & 53 & Casada & Vespertino & Posgrado \\
PE5 & Femenino & 52 & Casada & Nocturno & Posgrado \\
PE6 & Femenino & 45 & Casada & Nocturno & Posgrado \\
PE7 & Femenino & 33 & Soltera & Nocturno & Licenciatura \\
PE8 & Femenino & 44 & Soltera & Matutino & Licenciatura \\
PE9 & Masculino & 36 & Casado & Vespertino & Posgrado \\
PE10 & Femenino & 50 & Casada & Matutino & Licenciatura \\
\hline
\end{tabular}

Fuente: Cédula de datos personales. 


\section{1) Cuidado desde la perspectiva del supervisor} de enfermería: El cuidado representa la esencia de enfermería yse han desarrollado diversas definiciones para conceptualizar de manera integral el término, sin embargo, el SE lo relaciona mayormente con el cuidado al otro y no a sí mismo. Hay que tomar en cuenta que el cuidado es una forma de vida que engloba todas las esferas de la persona, incluyendo la espiritual y que, al desempeñarse como SE, el cuidado es dirigido hacia el personal operativo. Al respecto se preguntó a los participantes su concepto de cuidado:

"El cuidado engloba todas las esferas del ser incluyendo la mental y espiritual que es la que no cuidamos en las demás" (P01). "Es la razón de ser de enfermería, ver por el otro" (P02). "El cuidado se realiza de forma integral atendiendo las necesidades básicas del paciente"(P03).

Así mismo, se preguntó al SE cómo cuidan del personal operativo, ya que no están inmersos en el cuidado directo al paciente:

"Conforme pasa el tiempo conoces más a tu personal, tanto que con solo verlos a los ojos sabes que no están bien y les ofreces tu ayuda" (P10). "Ya que uno no está con los pacientes, nuestros clientes son los enfermeros operativos, al escuchar y satisfacer sus necesidades laborales e incluso personales cuando algo se ofrece es como los cuidamos" (P04). "El personal debe estar bien cuidado para brindar un cuidado de calidad al paciente hospitalizado, por lo tanto, es muy importante que nosotros cuidemos de ellos" (P09).

Es fundamental que el SE conozca al personal operativo para detectar sus necesidades, incluso a través del lenguaje no verbal y comprender que para cuidar de otras personas, se tiene que estar bien, primero, con uno mismo; no es posible brindar cuidado de calidad sin antes cuidarse como persona. Sin embargo, al detectar las necesidades de los demás no es capaz de visualizar las propias, descuidando así su esfera individual:

"Para cuidar bien de otros debemos cuidarnos a nosotros mismos primero, pero es lo último que hacemos, tenemos malos hábitos" (P03). "Nosotros como enfermeros siempre somos los últimos en cuidarnos porque siempre estamos cuidando a los demás"(P07).
2) Interacción entre el supervisor de enfermería y sus compañeros de trabajo: Como trabajador de la salud, el SE presenta necesidades que pueden influir en su desempeño laboral, entre ellas el ser escuchado y que les sean comunicadas sus áreas de oportunidad por parte de los superiores; así mismo, el reconocimiento a su trabajo fomentará la satisfacción laboral y la motivación intrínseca que tienen impacto directo sobre el desempeńo del personal operativo:

"El trabajador necesita que reconozcan su trabajo y aqui a nosotros no nos reconocen" (P08). "Falta mucha integración en el hospital, no nos hacen caso los superiores"(P03). "Ojalá los jefes tuvieran la apertura para escucharnos y retro-alimentarnos, pero también felicitarnos" (P05).

El cuidado al SE es percibido en función de la satisfacción del ambiente laboral y de las relaciones de trabajo que se establecen como equipo entre ellos y el personal operativo; al sentir satisfacción laboral, el personal operativo cuida del SE. Cuando se preguntó a los participantes si se sentían cuidados por el personal operativo, si recibían apoyo por parte del personal directivo y qué necesidades de cuidado tenían, estos respondieron:

"Si nos cuidan pero todo es únicamente lo que dicen las condicionesgenerales de trabajo"(P01). "Considero que para sentirme cuidada necesito cuidar a mi personal, hacer mancuerna con ellos y organizarnos, asi mis jefes me cuidan a mi también" (P05). "Si se mantiene un ambiente de trabajo saludable, los resultados se verán reflejados en el bienestar y productividad del personal de enfermería y asi todos estaremos mejor" (P07).

El cuidado requiere establecer un proceso de comunicación entre el SE y directivos de la institución de manera clara, efectiva y de confianza, para la correcta recepción del mensaje; en ocasiones no es así, el personal directivo no respeta la jerarquía y funciones del SE, olvidando la toma de decisiones consensuadas. Se preguntó sobre la importancia de la comunicación en el entorno laboral y cómo la perciben entre los compañeros de trabajo:

"Escomún que el personalsienta desconfianza alpedirte cualquier cosa, ya que todos los supervisores tenemos diferentes formas de expresarnos" (P03). "Se realizan reuniones con los directivos para tomar decisiones y nos 
invitan, pero no toman en cuenta nuestra opinión" (P09). "Creo que hay que fortalecer los canales de comunicación y no omitir ningún mensaje importante para estar todos en la misma sintonía" (P10).

\section{3) Desarrollo profesional como parte del} cuidado al supervisor de enfermería: Entre las necesidades de cuidado del SE se encuentran las oportunidades para el desarrollo profesional y el plan de carrera que ofrece cada institución, inherente a la satisfacción laboral, se traduce en crecimiento personal. La mayoría de las veces los puestos de mayor jerarquía no se ofrecen de acuerdo a capacidades de las personas, sino a intereses de los directivos, perjudicando así al SE debido a que no se sienten protegidos o cuidados por los superiores: "Es importante que haya crecimiento, que nos dejen estudiar y seguirnos superando" (P10). "Cada vez que nos cambian de director del hospital nos llega la incertidumbre sobre nuestro futuro, ya que ellos traen a su gente" (P01). "Yo creo que cada vez que el director asigne puestos debe ser con base en un examen $y$ entrevistas para conocer sus aptitudes y actitudes" (P04).

La falta de capacitación al puesto hace que el SE se perciba así mismo como un profesionista vulnerable y por ende, disminuya su satisfacción laboral y la del equipo de trabajo; parte de las actividades de cuidado profesional implican ayudar a la persona para que desarrolle al máximo su potencial:

"Cuando me asignaron al puesto no tuve una inducción y el primer mes me fue muy mal, cometi muchos errores pero poco a poco he ido aprendiendo" (P08). "Alprincipio cuando comencé en supervisión me sentía muy vulnerable pero mi compañera era nueva en el puesto también y la veía a ella más vulnerable" (P09). "El primer día en supervisión llegué y sentí que no sabia nada" (P03). "Al cuidar, ayudamos a las personas a que desarrollen su potencial, al no capacitarnos en el puesto no nos cuidaron"(P06).

Los puestos de supervisión conllevan sacrificios personales y es común que no se respete el horario laboral, los días de descanso o, incluso, los períodos de comidas. Estas situaciones perjudican la salud mental del SE pues no se cuidan ni satisfacen sus necesidades primordiales por la institución, lo que deriva en un constante estrés dentro del trabajo. Al respecto los participantes comentan:

"Cuando entras a supervisión requieres estar disponible las 24 horas del día por teléfono y eso me estresa mucho" (P03). "El puesto de supervisión me gusta mucho, es crecimiento profesional pero muy estresante" (P02). "En muchas ocasiones nos tenemos que quedar horas después de nuestra salida y eso es restar tiempo personal" (P07). "Hace mucho que no sé lo que es mi hora de comida, a veces ni mis descansos por el exceso de trabajo"(P09).

\section{DISCUSIÓN}

Se considera que el diseño de estudio fue apropiado para obtener información detallada y cumplir con el objetivo. La investigación cualitativa permite conocer a los actores cuando se desarrollan en su propio contexto y facilita el rapport necesario para establecer relaciones de confianza, lo que se visualiza cuando se realizó la invitación al estudio y todos los participantes accedieron a colaborar. Lo anterior puede estar influenciado porque la mayoría de los participantes contaba con estudios de posgrado y la evidencia señala que cuando el personal de enfermería cuenta con estudios de maestría, desarrolla mejor las competencias para trabajar con otros miembros del equipo de salud y utiliza el pensamiento crítico para la investigación y gestión ${ }^{(16)}$.

De acuerdo con los resultados de esta investigación, el SE conceptualiza el cuidado como la razón de ser de enfermería, sin embargo, no menciona el autocuidado como un recurso para sí mismo. El cuidado se ha convertido en la esencia de la disciplina y se observa al cuidar del paciente y sus familiares de manera holística, satisfaciendo sus necesidades ${ }^{(17)}$; también cuando el personal de enfermería realiza acciones de autocuidado cuyos resultados se reflejan no solo en su persona, sino también en el equipo de trabajo y los pacientes, pero al parecer, fomentar los espacios de trabajo como un espacio protector parece una tarea difícil ${ }^{(18-20)}$.

El SE menciona que otorga cuidado al personal de enfermería operativo cuando al conocer sus necesidades, a través de la comunicación y el lenguaje no verbal, les ofrece apoyo laboral y personal; con ello también se proporciona cuidado de manera indirecta a los pacientes. En efecto, cuando los líderes de enfermería fomentan un 
ambiente de profesionalismo, que incluye el trabajo colaborativo, la toma de decisiones consensuadas y además se permite el desarrollo profesional de los colaboradores, se promueve el liderazgo transformacional, mismo que se relaciona con el incremento de la calidad del cuidado ${ }^{(21,22)}$. El liderazgo transformacional es uno de los estilos más importantes en enfermería, debido a que ayuda a motivar al personal y a conectarse con él para percibir sus necesidades, cuestiones claves que se asocian, de manera importante, a la satisfacción laboral ${ }^{(23)}$.

Cuando se adopta un estilo de liderazgo transformacional, se influye de manera positiva en el personal y por consiguiente en el éxito de la institución, posicionándose la comunicación como su principal atributo. Relacionado con esto, se menciona que proporcionar retroalimentación al personal influye en la permanencia del mismo y en el cumplimiento de los objetivos laborales ${ }^{(24)}$.

Para que la información sea transmitida en sus diferentes canales a través del emisor y receptor, es esencial la buena comunicación, más aún en aquellos escenarios en los que interaccionan las personas ${ }^{(25)}$. Aun así, en este estudio, los participantes comentan que una de las principales debilidades que se percibe en su puesto es la falta de comunicación o que no se utilizan los canales adecuados para la misma, y que el mensaje que se quiere transmitir no llega al receptor, principalmente en la interacción con sus superiores.

Los resultados de este estudio muestran que el SE no se siente satisfecho con la forma en que el personal directivo asigna los puestos de supervisión y que no existe una selección basada en competencias, ni se brinda capacitación para asumir el puesto. Frente a esta situación, es importante señalar que el reemplazo generacional de líderes en enfermería es de suma importancia para continuar con el cumplimiento de los objetivos institucionales. En ocasiones, dicho proceso no se realiza de manera adecuada y se asigna como supervisor a personal nuevo o que ha tenido poco contacto con sus superiores y, por ende, desconocen el objetivo del puesto y la dinámica de los departamentos del hospital. Superar esta realidad implica planificar la sucesión y desarrollar liderazgo, a objeto de mejorar el ambiente laboral y el cuidado de enfermería ${ }^{(26,27)}$.

Si el ambiente laboral es determinante para que el SE decida continuar o renunciar a su puesto, entonces resulta importante cuidar de este profesional por el rol que desempeña como gestor del cuidado y líder del personal operativo. Dentro de las principales razones para renunciar a la supervisión se encuentran: la insatisfacción laboral, falta de reconocimiento a su trabajo y la percepción de que sus superiores no son empáticos con ellos ni cuidan de sus necesidades como personas y trabajadores, por lo que es necesario que el personal directivo, en las instituciones de salud, fomente un buen ambiente laboral para cuidar del $\mathrm{SE}^{(28)}$.

Cabe señalar que el estudio presentó algunas limitaciones derivadas del trabajo y del tipo de institución en donde se realizó el estudio. Por la carga de trabajo que tenían los participantes fue difícil que se ausentaran durante su jornada laboral para las entrevistas, pero fue la opción elegida porque en su mayoría rehusaron participar fuera del horario de trabajo. De igual forma, el estudio se desarrolló únicamente en el contexto de dos instituciones públicas de segundo nivel en el norte de México, pero para comprender mejor el fenómeno de estudio se recomienda que futuras investigaciones exploren otras áreas e incorporen otras instituciones y se obtenga información del SE que labora en hospitales privados.

\section{CONCLUSIONES}

El SE percibe el ambiente laboral a través de tres atributos: el cuidado, la interacción entre compañeros de trabajo y el desarrollo profesional. El SE conceptualiza el cuidado como una actividad dirigida a otros pero no para sí mismo.

La comunicación efectiva y estilo de liderazgo desempeñan una función importante para la interacción entre el personal de enfermería operativo, el SE y el personal directivo de las instituciones, pero estos atributos no se perciben de manera adecuada por ellos.

La mayor parte de los supervisores de enfermería no cuentan con antecedentes en el área operativa, ni reciben capacitación o inducción a la asignación del puesto, por lo que al convertirse en supervisores se les dificulta realizar ciertas funciones, como la toma de decisiones y la relación con el personal operativo. 


\section{REFERENCIAS}

1. Huber D. Leadership and nursing care management. 6ta ed. Iowa: Saunders; 2017. 544 p.

2. Yoder-Wise P. Leading and managing in Nursing. 7a ed. Texas: Mosby; 2019. 591 p.

3. American Organization of Nurse Executives [Internet]. Chicago, IL: AONE Nurse Manager Competencies. 2015 [citado 8 ene 2020]. Disponible en: https://www.nurseleader.com/article/S15 41-4612(05)00007-8/fulltext

4. Guerra S, Prochnow A, Trevizan M, Guido L. El conflicto en el ejercicio gerencial del enfermero en el ámbito hospitalario. Rev Latino-Am Enfermagem [Internet]. 2011 [citado 8 ene 2020]; 19(2): 1-8. Disponible en: http://www.scielo.br/pdf/rlae/v19 n2/es_19.pdf

5. Spano-Szekely L, Quinn GM, Clavelle J, Fitzpatrick J. Emotional Intelligence and Transformational Leadership in Nurse Managers. J Nurs Adm [Internet]. 2016 [citado 3 feb 2020]; 46(2): 101108. Disponible en: https://www.ncbi.nlm.nih. gov/pubmed/26796823

6. Kunie K, Kawakami N, Shimazu A, Yonekura Y, Miyamoto Y. The relationship between work engagement and psychological distress of hospital nurses and the perceived communication behaviors of their nurse managers: A cross-sectional survey. Int J Nurs Stud [Internet]. 2017 [citado 27 ene 2021]; 71: 115-124. Disponible en: https:// www.sciencedirect.com/science/article/abs/pii/ S0020748917300846

7. Rouse R, Al-Maqbali M. Identifying nurse managers' essential communication skills: an analysis of nurses' perceptions in Oman. J Nurs Manag [Internet]. 2014 Mar 22 [citado 3 feb 2020]; (2): 192-200. Disponible en: https://www. ncbi.nlm.nih.gov/pubmed/24529216

8. Feather R, Ebright P, Bakas T. Nurse Manager Behaviors that RNs Perceive to Affect their Job Satisfaction. Nurs Forum [Internet]. 2015 Abr [citado 23 ene 2020]; 50 (2): 125-136. Disponible en: https://www.ncbi.nlm.nih.gov/pubmed/24428342

9. Iglesias-Armenteros AL, Torres-Esperón JM. Intervención para la mejora del clima organizacional en enfermería. Infodir [Internet]. 2019 Ene [citado 25 ene 2020]; 28: 98-108. Disponible en: https:// www.medigraphic.com/pdfs/infodir/ifd-2019/ ifd1928i.pdf

10 Semper A, Sánchez Z, Segredo A, Hernández A, Álvarez M, Mestre V. Clima organizacional en la atención a pacientes con morbilidad materna extremadamente grave. Rev cub salud pública [Internet]. 2019 Ene [citado 27 ene 2020]; 45(1): e988. Disponible en: http://scielo.sld.cu/scielo. php?pid=S0864-34662019000100002\&script + sci_arttext\&tlng=pt

11. Parchment J, Andrews D. The Incidence of Workplace Bullying and Related Environmental Factors Among Nurse Managers. J Nurs Adm [Internet]. 2019 Mar [citado 19 ene 2020]; 49(3): 132-137. Disponible en: https://www.ncbi.nlm. nih.gov/pubmed/30789556

12. Grubaugh M, Flynn L. Relationships Among Nurse Manager Leadership Skills, Conflict Management, and Unit Teamwork. J Nurs Adm [Internet]. 2018 Jul [citado 28 ene 2020]; 48(7-8): 383-388. Disponible en: https://www.ncbi.nlm. nih.gov/pubmed/30028814

13. Hernández R, Mendoza C. Metodología de la investigación. Las rutas cuantitativa, cualitativa y mixta. 1er ed. México: McGraw-Hill; 2018. 752 p.

14. Grove S, Gray J, Burns N. Investigación en enfermería. 6ta ed. España: Elsevier; 2016. 544 p.

15. México. Reglamento de la Ley General de Salud en Materia de Investigación para la Salud. Secretaría de Salud. 2018. Disponible en: http://www.salud. gob.mx/unidades/cdi/nom/compi/rlgsmis.html

16. Wangensteen S, Finnbakk E, Adolfsson A, Kristjansdottir G, Roodbol P, Ward H et al. Postgraduate nurses' self-assessment of clinical competence and need for further training. A European crosssectional survey. Nurse Educ Today [Internet]. 2018 Mar [citado 29 ene 2020]. Disponible en: https://www.ncbi.nlm.nih.gov/pubmed/29306748

17. Tanaka M, Taketomi K, Yonemitsu Y, Kawamoto R. Professional behaviours and factors contributing to nursing professionalism among nurse managers. J Nurs Manag [Internet]. 2016 Ene [citado 26 ene 2020]; 24(1): 12-20. Disponible en: https://www. ncbi.nlm.nih.gov/pubmed/25355449.

18. Macaya B, Aranda F. Cuidado y autocuidado en el personal de salud: enfrentando la pandemia COVID-19. Rev Chil Anest [Internet]. 2020 [citado 22 ene 2021]; 49(3): 356-362. Disponible en: https://revistachilenadeanestesia.cl/revchilanest v49n03-014/

19. Andrews H, Tierney S, Seers K, Wand T, Fraser J. Needing permission: The experience of self-care and self-compassion in nursing: A constructivist grounded theory study. Int J Nurs Stud [Internet]. 2020 Abr [citada 26 ene 2021]; 101: 103436. Disponible en: https://pubmed.ncbi.nlm.nih.gov/ 31670223/

20. Richards K. Self-care is a lifelong journey. Nurs Econ [Internet]. 2013 Jul [citada feb 2020]; 31(4): 198-202. Disponible en: https://pdfs. semanticscholar.org/bdd6/c908e9259ddd3458b41 7187299f3860fe486.pdf 
21. Cullen JG. Nursing management, religion and spirituality: a bibliometric review, a research agenda and implications for practice. J Nurs Manag [Internet]. 2016 Abr [citado feb 2020]; 24(3): 291-299. Disponible en: https://www.ncbi.nlm. nih.gov/pubmed/26496849

22. Wong C. Connecting nursing leadership and patient outcomes: state of the science. J Nurs Manag [Internet]. 2015 Abr [citado 3 feb 2020]; 23 (3): 275-278. Disponible en: https://onlinelibrary. wiley.com/doi/full/10.1111/jonm.12307

23. Perilla-Toro L, Gómez-Ortiz V. Relación del estilo de liderazgo transformacional con la salud y el bienestar del empleado: el rol mediador de la confianza en el líder. Rev psicol trab org [Internet]. 2017 Ago [citado 23 ene 2021]; 33(2): 95-108. Disponible en: http://scielo.isciii.es/pdf/rpto/v33 n2/1576-5962-rpto-33-02-00095.pdf

24. Manning J. The Influence of Nurse Manager Leadership Style on Staff Nurse Work Engagement. J Nurs Adm [Internet]. 2016 Sep [citado 7 feb 2020]; 46(9): 438-443. Disponible en: https:// www.ncbi.nlm.nih.gov/pubmed/27496584
25. Hernández-Mendo A, Fernández DMM, Raimundi M. Un cuestionario para evaluar los canales de comunicación. Revista iberoamericana de psicología del ejercicio y el deporte [Internet]. 2015 [citado 6 feb 2020]; 12(1): 33-41. Disponible en: https:// www.redalyc.org/html/3111/311148817003

26. Gomes A, Bouttelet D, Miranda L, Queiroz AL, Pedreschi L. Expectativa de enfermeros sobre la sucesión de liderazgos en el contexto hospitalario. Rev Latino-Am Enfermagem [Internet]. 2019 [citado 23 ene 2021]; 27: e3178. Disponible en: http://dx.doi.org/10.1590/1518-8345.2833.3178.

27. Dyess S, Sherman R, Pratt B, Chiang-Hanisko L. Growing Nurse Leaders: Their Perspectives on Nursing Leadership and Today's Practice Environment. Online J Issues Nurs [Internet]. 2016 Ene [citado 9 feb 2020]; 14(21): 7. Disponible en: https://www.ncbi.nlm.nih.gov/pubmed/27853273

28. Hewko S, Brown P, Fraser K, Wong C, Cummings G. Factors influencing nurse managers' intent to stay or leave: a quantitative analysis. J Nurs Manag [Internet]. 2015 Nov [citado 9 feb 2020]; 23(8): 1058-1066. Disponible en: https://www.ncbi.nlm. nih.gov/pubmed/25491021 